\title{
Chromatin structure and transposable elements in organismal aging
}

\author{
Jason G. Wood* and Stephen L. Helfand \\ Department of Molecular Biology, Cell Biology, and Biochemistry, Brown University, Providence, RI, USA
}

\section{Edited by:}

Philipp Oberdoerffer, National Cancer Institute, USA

\section{Reviewed by:}

Philipp Oberdoerffer, National Cancer Institute, USA

Karim Mekhail, University of Toronto, Canada

Patrick Maxwell, Rensselaer

Polytechnic Institute, USA

\section{${ }^{*}$ Correspondence:}

Jason G. Wood, Department of

Molecular Biology, Cell Biology, and

Biochemistry, Brown University,

Box G-L163, 185 Meeting Street

Providence, RI 02912, USA

e-mail: jason_wood@brown.edu
Epigenetic regulatory mechanisms are increasingly appreciated as central to a diverse array of biological processes, including aging. An association between heterochromatic silencing and longevity has long been recognized in yeast, and in more recent years evidence has accumulated of age-related chromatin changes in Caenorhabditis elegans, Drosophila, and mouse model systems, as well as in the tissue culture-based replicative senescence model of cell aging. In addition, a number of studies have linked expression of transposable elements (TEs), as well as changes in the RNAi pathways that cells use to combatTEs, to the aging process. This review summarizes the recent evidence linking chromatin structure and function to aging, with a particular focus on the relationship of heterochromatin structure to organismal aging.

Keywords: chromatin, heterochromatin, transposable elements, retrotransposons, RNAi, histone modifications, epigenetics of aging, silencing

\section{INTRODUCTION}

In addition to the genetic information encoded in the nuclear DNA genome, cells are also able to store information in the form of epigenetic modifications to proteins and nucleic acids that affect cellular processes and phenotypes. Some of these epigenetic mechanisms include methylation, acetylation, and other covalent modifications of histones and other proteins, methylation of the DNA itself, and posttranscriptional regulation of both coding and non-coding RNA species. In recent years, epigenetic regulation has begun to be recognized as playing a key role in a host of biological processes, including aging. Unlike genetic changes such as base pair deletion or mutation, epigenetic changes are generally reversible and thus represent not only an easy mechanism for cells to regulate various processes, but also an attractive therapeutic target for potential intervention in human disease states.

Many of the most prominent epigenetic modifications, including covalent post-translational modification of histones and chromatin remodeling, take place in the context of chromatin. The basic structure of chromatin consists of DNA wrapped around a histone octamer core, forming a nucleosome. The repeating nucleosome units are then packaged together and condensed in a complex higher order structure. The highly conserved core histone proteins (histones $\mathrm{H} 2 \mathrm{~A}, \mathrm{H} 2 \mathrm{~B}, \mathrm{H} 3$, and $\mathrm{H} 4$ ) contain $\mathrm{N}$-terminal tails that are rich in lysine residues, which are prime targets for a number of covalent posttranslational modifications, including acetylation, methylation, and phosphorylation. Modification of these residues creates a "histone code" that serves a number of important regulatory functions, including recruitment of transcription factors and polymerases, and assembly of chromatin complexes that either promote or repress transcription. Histone marks are added and removed by a host of methyl- and acetyltransferases, and deacetylases and demethylases, which frequently have catalytic activity specific to a single residue. Chromatin structure is not uniform throughout the genome, and is usually classified into two types: euchromatin and heterochromatin. Euchromatin is generally transcriptionally active, gene rich, accessible to polymerases and transcription factors, and enriched for "active" histone marks such as H3K4 and H3K36 methylation and H4K16 acetylation. Heterochromatin domains, on the other hand, are normally gene poor, transcriptionally repressed, tightly packed and inaccessible to transcription factors, and enriched for "repressive" histone marks such as H3K9 or H3K27 methylation.

In this review we highlight evidence from the recent literature linking changes in chromatin structure and function to the aging process. We discuss early theories of chromatin change with age, highlighting pioneering work in the yeast S. cerevisiae. We also discuss the role of chromatin in the replicative senescence model of cellular aging. We then examine evidence for chromatin changes in aging in animal model systems, including Caenorhabditis elegans, D. melanogaster, mice, and rats. We discuss the important regulatory role of the spatial organization of chromatin within the nucleus, and how it may contribute to aging both at the organismal and cellular level. Finally, we review the link between chromatin and transposable elements, and discuss how changes in expression and activity of transposable elements may be relevant to the aging process.

\section{HETEROCHROMATIN LOSS MODEL OF AGING}

It has long been supposed that epigenetic effects influence the aging process (Macieira-Coelho, 1991; Kennedy et al., 1995). To date there have been a number of studies looking at how the structure of chromatin changes as organisms age (Oberdoerffer and Sinclair, 2007). Much of the early work linking chromatin to aging was done in the budding yeast $S$. cerevisiae. Because yeast is a 
single-celled organism, one of the ways aging is measured in this organism is to assay replicative lifespan, or the number of daughter cells each new mother cell can produce by budding before entering a non-dividing senescent state. In experiments performed in the 1990s, it was observed that aging and consequent sterility in yeast was correlated with a loss of characteristic heterochromatic silencing at telomeres, mating type locus, and ribosomal DNA (rDNA) repeats (Kim et al., 1996; Smeal et al., 1996; Kennedy et al., 1997). The association between chromatin and aging was strengthened when Sir2, which had originally been identified in a screen for factors involved in heterochromatic genetic silencing, was found to be associated with a longevity phenotype in budding yeast (Kaeberlein et al., 1999). Sir2 was subsequently characterized enzymatically as a $\mathrm{NAD}^{+}$-dependent histone deacetylase (Frye, 2000; Imai et al., 2000; Landry et al., 2000), and later the sirtuin family (Sir2 homologues) was found to have a wide range of activities and targets (reviewed in Haigis and Sinclair, 2010). This early yeast work lent support to the heterochromatin loss model of aging, where heterochromatin structure within the genome breaks down or weakens as an organism or cell ages (Villeponteau, 1997; Tsurumi et al., 2012). The loss of heterochromatin is expected to have deleterious effects on cellular homeostasis, for instance by causing aberrant expression of normally repressed genes, or redirection of limited energy toward repair or maintenance of damaged heterochromatin regions. Inappropriate transcription of silenced genes may cause cellular damage through a number of different mechanisms. An increase in transcriptional noise may cause cumulative harm by appropriating cellular factors necessary for transcribing and expressing normal genes. Alternatively, loss of silencing may lead to expression of certain genes that are causative of aging phenotypes, such as in yeast when silent mating cassette derepression leads to co-expression of both mating type genes, causing sterility and senescence (Smeal et al., 1996). However, the specific consequences of loss of heterochromatin silencing and the potential role of the transcriptional and translational machinery in downstream effects remain poorly characterized.

\section{CHROMATIN STRUCTURE IN YEAST AGING}

In more recent years, a number of yeast studies have looked at changes in histone structure and histone post-translational modifications with age (reviewed in Feser and Tyler, 2011). In yeast cells, levels of H4K16 acetylation increase with age, and this is accompanied by a decrease in Sir2 levels (Sir2 deacetylates H4K16ac; Dang et al., 2009). In this study, aging was also associated with a loss of histones in certain heterochromatic regions, and a concomitant decrease in silencing at these loci. In addition, a study by Feser et al. found that yeast cells lacking the histone chaperone Asf1 had a shortened lifespan, and this was due to a defect in histone $\mathrm{H} 3 \mathrm{~K} 56$ acetylation and a failure to properly regulate histone levels (Feser et al., 2010). This study also reported that histone protein levels themselves decline with age. Interestingly, when histone levels were raised artificially, either by knocking out HIR1, which represses histone transcription, or by overexpressing histones $\mathrm{H} 3$ and $\mathrm{H} 4$ directly, lifespan was increased. Together with earlier work, these studies support a strong link between aging and chromatin structure in yeast, and suggest that aging results at least in part from a failure to maintain proper chromatin structure with time.

\section{CHROMATIN IN REPLICATIVE SENESCENCE}

The link between chromatin structure and aging has also been examined in the replicative senescence of tissue culture cells. Cells in culture will divide a finite number of times before ceasing mitosis and entering into a non-dividing quiescent state, a process termed replicative senescence. In a way, experiments in this system are akin to the measurement of replicative lifespan commonly performed in budding yeast. A number of age-related changes in chromatin structure have been observed in senescent cells (O'Sullivan and Karlseder, 2012). Histone levels are known to decline during replicative senescence, and several histone methylation and acetylation marks redistribute in senescent cells (O'Sullivan et al., 2010; Ivanov et al., 2013). As cells senesce, they also form regions of dense non-pericentromeric heterochromatin termed senescence-associated heterochromatin foci (SAHF), which are characterized by a high level of repressive heterochromatin histone marks such as $\mathrm{H} 3 \mathrm{~K} 9 \mathrm{me} 3$ and $\mathrm{H} 3 \mathrm{~K} 27 \mathrm{me} 3$ (Narita et al., 2003; Zhang et al., 2005; Kosar et al., 2011; Chandra et al., 2012). A recent study by Shah et al. examined large scale chromatin changes in senescent cells with a ChIP-seq approach (Shah et al., 2013). When compared with proliferating cells, in senescent cells both $\mathrm{H} 3 \mathrm{~K} 4 \mathrm{me} 3$ and $\mathrm{H} 3 \mathrm{~K} 27 \mathrm{me} 3$ were enriched in large contiguous regions that correlated with lamin $\mathrm{B} 1$-associated domains (LADs). Areas of contiguous negative enrichment of H3K27me3 were also observed and tended to be rich in genes and promoters. Interestingly, senescence associated changes in these histone marks were also correlated with senescence associated gene expression changes, with loss of $\mathrm{H} 3 \mathrm{~K} 4 \mathrm{me} 3$ at down-regulated genes, and loss of H3K27me3 at up-regulated genes (Shah et al., 2013).

De Cecco et al. (2013) examined replicatively senescent cells using the FAIRE ( formaldehyde-assisted isolation of regulatory elements) technique (Giresi and Lieb, 2009), which measures open (transcriptionally active) and closed (transcriptionally silent) chromatin states. The technique exploits the differential partitioning during phenol/chloroform extraction of DNA fragments containing densely packed histones (more protein, more likely to be in organic phase) or relatively sparse histones (more DNA, more likely to be in aqueous phase). They observed that replicatively senescent cells tend to show a "smoothing" of chromatin relative to dividing cells (De Cecco et al., 2013). In other words, regions of open and closed chromatin are less distinct and look more similar to each other in senescent cells. Promoters and enhancers of genes that are active in dividing cells become more closed upon senescence, and regions of normally silent heterochromatin become more open. Results from these replicative senescence studies suggest that structural changes in chromatin with age are an important conserved characteristic in metazoans.

\section{AGE AND CHROMATIN IN ANIMAL MODEL SYSTEMS C. elegans}

In addition to evidence accumulating from yeast and cellular senescence models of aging, several studies in animal model systems have assessed changes in chromatin structure during organismal aging. In the roundworm C. elegans, a number of recent studies have demonstrated a relationship between lifespan 
and certain histone modifications. Disruption of the ASH-2 complex in worms, which contains a H3K4 methyltransferase, led to an increase in worm lifespan (Greer et al., 2010). Conversely, disruption of RBR-2, a H3K4 demethylase, led to a reduced lifespan and supported the conclusion that excessive H3K4 trimethylation, a hallmark of actively transcribed chromatin, was detrimental to lifespan. A follow up study showed that the effects of extended lifespan induced by these genetic modifications were heritable up to the third generation (Greer et al., 2011).

In addition to changes in active chromatin marks such as $\mathrm{H} 3 \mathrm{~K} 4 \mathrm{me} 3$, there have also been reports of associations between aging and heterochromatin marks. Knockdown of the worm ortholog of LSD1, a histone demethylase that targets methylated $\mathrm{H} 3 \mathrm{~K} 4$ and $\mathrm{H} 3 \mathrm{~K} 9$ residues, led to a significant increase in lifespan (McColl et al., 2008). The H3K27me3 mark is found in association with regions of Polycomb heterochromatin, a type of repressive heterochromatin responsible for regulating silencing of numerous genes during development. A pair of studies examined the role of H3K27 methylation in worm aging, and found that disruption of the H3K27me3 demethylase UTX-1 led to increased levels of $\mathrm{H} 3 \mathrm{~K} 27 \mathrm{me} 3$ marks in the genome as well as increased lifespan (Jin et al., 2011; Maures et al., 2011). Normal aging also showed an increase in UTX-1 activity, as well as a decline in $\mathrm{H} 3 \mathrm{~K} 27 \mathrm{me} 3$ methylation. The lifespan extension was dependent on a functional daf-16 gene, suggesting the effect functions through the insulin signaling pathway. Together, these $C$. elegans studies showed that aging is associated with a number of histone changes, and that genetic intervention to directly modulate histone marks can lead to changes in lifespan.

\section{Drosophila melanogaster}

The fruit fly Drosophila melanogaster has also been used as a model system to study chromatin and aging. A whole genome study of aging fly heads showed several chromatin changes that occurred with age (Wood et al., 2010). Marks typifying active chromatin, such as H3K4me3 and H3K36me3, showed a general decline across genes with age. However, the most notable change was a significant decrease with age in the enrichment of the repressive heterochromatin mark $\mathrm{H} 3 \mathrm{~K} 9 \mathrm{me} 3$ as well as HP1 (heterochromatin protein 1, a component of heterochromatin) at pericentric heterochromatin loci. Changes in nuclear localization and organization of both $\mathrm{H} 3 \mathrm{~K} 9 \mathrm{me} 3$ and $\mathrm{HP} 1$ were also observed. Additionally, genes that lost H3K9me3 or HP1 with age trended toward increased expression. In another recent study, Larson et al. genetically manipulated expression of HP1, and observed that flies with decreased HP1 expression exhibited shortened lifespan (Larson et al., 2012). Conversely, flies overexpressing a transgenic HP1 showed an increase in lifespan (Larson et al., 2012), although an earlier study using a free duplication containing the HP1 locus failed to observe lifespan extension (Frankel and Rogina, 2005). Flies with decreased HP1 also showed premature muscle degeneration compared with controls, while HP1 overexpressing flies showed increased muscle function and structure with age (Larson et al., 2012). In addition, old flies showed an overall decrease in heterochromatin as well as nuclear reorganization of HP1. Flies with decreased HP1 expression also showed a dramatic increase in ribosomal RNA (rRNA) transcripts, while flies overexpressing HP1 had a decline in such transcripts, demonstrating that disruption of heterochromatinstructure is sufficient to modulate transcription from the repetitive rDNA locus. These Drosophila data fit well with the heterochromatin loss model of aging described above, whereby loss of repressive heterochromatin leads to loss of silencing and aberrant gene expression, with consequent deleterious effects on the cell or organism. However, not all reported studies are consistent with this model. In contrast to the worm H3K27me3 studies described above (Jin et al., 2011; Maures et al., 2011), a study examining genetic mutations in the H3K27 histone methyltransferase component of the Polycomb complex in Drosophila reported that loss of function of this gene led to a decrease in H3K27 methylation, as well as an increase in lifespan (Siebold et al., 2010). These sometimes conflicting results underscore the possibility that there may be important tissue specific differences or differences between species in how chromatin marks are processed and their consequent effects on aging.

\section{MAMMALS}

Age-related chromatin changes have also been observed in mammalian model systems, primarily mouse and rat. Most mammalian studies have tended to focus on changes in histone acetylation with age. A recent study of chromatin structure with age in the mouse cochlea showed a decrease in histone acetylation and an increase in histone methylation in aged mice compared to young mice (Watanabe and Bloch, 2013). Notable differences in DNA methylation patterns have also been observed between young and old mice, and these methylation changes are consistent across a number of different tissue types (Maegawa et al., 2010). In addition to direct observation of epigenetic marks in aging tissue, several studies have identified a role for histone modifications in functional and behavioral studies of mouse aging. Using a neurodegeneration mouse model allowing for targeted induction of neuronal loss, Fischer et al. examined the molecular basis for the environmental enrichment (EE) paradigm that is known to increase synaptic function and memory formation (Fischer et al., 2007). In this study, EE led to a significant increase in learning and memory among mice that had undergone neuronal loss, and this was correlated with an increase in histone acetylation and methylation at a number of different residues on histones H3 and H4. Furthermore, treatment with sodium butyrate, a histone deacetylase inhibitor, was sufficient to increase learning and memory in these mice (Fischer et al., 2007). In a similar study, this time examining age-related cognitive decline rather than induced neurodegeneration, it was observed that the brains of aged mice when compared with younger mice exhibited a hypoacetylation of H4K12 in the hippocampus, with a concomitant failure to activate a gene expression program necessary for memory consolidation (Peleg et al., 2010). When aged mice were treated with a histone deacetylase inhibitor in the hippocampus, H4K12 acetylation patterns, memory associated gene expression profiles, and learning behavior were all restored. Similarly, a study of aging rat brains showed reduced histone acetylation of $\mathrm{H} 3 \mathrm{~K} 9$ and $\mathrm{H} 4 \mathrm{~K} 12$ in the hippocampus with age, resulting in compromised expression of genes important for long-term potentiation (LTP; Zeng et al., 2011). Again, when rats were treated with histone deacetylase inhibitors, 
both acetylation levels as well as age-related LTP declines showed improvement.

Chromatin structure has also been implicated in mouse models of age-associated disease. In the APP/PS1 mouse model of Alzheimer's disease, a decrease in histone $\mathrm{H} 4$ acetylation as well as defects in LTP and memory formation was observed when compared to control mice (Francis et al., 2009). Interestingly, treatment with histone deacetylase inhibitors restored acetylation levels as well as cognitive function in the affected mice. A second study in this model confirmed that treatment with histone deacetylase inhibitors was able to correct age-related memory impairment (Kilgore et al., 2010). In a mouse model of Hutchinson-Gilford progeria, which shows defective lamin A processing and an associated short lifespan, H4K16 is hypoacetylated, and this is also observed during normal aging (Krishnan et al., 2011). Furthermore, either overexpression of the histone acetyltransferase Mof or chemical inhibition of histone deacetylase activity caused amelioration of age-related symptoms and extension of lifespan in this short-lived model.

While most of the mammalian model system data has tended to show a decrease in histone acetylation with age, as noted above Feser et al. observed an increase with age of the H4K16ac mark in yeast (Feser et al., 2010). In yeast, the H4K16ac mark is particularly associated with the mating type loci and subtelomeric regions that are normally silenced by the SIR complex, where Sir2 acts to deacetylate H4K16 (Millar and Grunstein, 2006). It is likely that the observed increase in H4K16 acetylation is indicative of the loss of silencing in these regions that is known to take place with age (Kennedy et al., 1997). In multicellular organisms, the situation is likely significantly more complex, and there may also be cell type or tissue specific effects that lead to the apparently contradictory results. Importantly, the identification of age-related chromatin changes in not only replicative senescence models such as yeast and tissue culture, but also in animal models of organismal aging, argues strongly for chromatin structure playing an important conserved role in regulating the aging process.

\section{SPATIAL ORGANIZATION OF CHROMATIN}

Chromatin is organized into discrete domains of euchromatin and heterochromatin within the linear genome. However, the spatial organization of chromatin within the nucleus also has important ramifications for regulation of gene expression (for recent reviews see Oberdoerffer and Sinclair, 2007; Bickmore and van Steensel, 2013; Gibcus and Dekker, 2013). It has long been appreciated that euchromatin and heterochromatin are not distributed equally throughout the volume of the nucleus. In yeast, heterochromatic telomere regions are preferentially associated with the nuclear envelope (Gotta et al., 1996). Furthermore, silencing of yeast chromatin can be switched on and off by targeting it to or away from the nuclear periphery (Andrulis et al., 1998; Feuerbach et al., 2002). In higher eukaryotes, chromatin regions termed lamin-associated domains (LADs) are similarly localized to the nuclear periphery, where they interact with the nuclear lamina. These LADs tend to be heterochromatic, gene poor, and refractory to transcription, and have been characterized in both mammalian as well as Drosophila cells (Pickersgill et al., 2006; Guelen et al., 2008). Interestingly, movement of these LADs away from the nuclear periphery is correlated with altered gene expression patterns (Peric-Hupkes et al., 2010). In addition to heterochromatin organization, transcriptionally active euchromatic regions are also located in distinct spatial domains within the nucleus known as transcription factories (Fraser and Bickmore, 2007; Rieder et al., 2012; Gibcus and Dekker, 2013).

Changes in nuclear architecture and spatial organization of chromatin have also been linked to both cellular senescence and organismal aging. Hutchinson-Gilford Progeria Syndrome (HGPS) is a rare genetic disorder characterized by symptoms that resemble premature aging, and is caused by mutations in the LMNA gene, which encodes lamin A, a key component of the nuclear lamina. These mutations lead to alternative splicing and incorrect processing of lamin $\mathrm{A}$, resulting in a dominant negative form of lamin A called progerin that disrupts proper nuclear architecture, leading to misshapen nuclei, changes in heterochromatin organization, and defects in DNA replication and transcription (Eriksson et al., 2003; Goldman et al., 2004). Phenotypes similar to those observed in HGPS have also been observed in normal aging in fibroblasts taken from older individuals. These age-related changes include altered lamin A processing and nuclear structure (Scaffidi and Misteli, 2006; McClintock et al., 2007). In addition, expression of the nuclear lamin B1 decreases naturally as cells enter senescence (Shimi et al., 2011; Freund et al., 2012; Shah et al., 2013). Furthermore, in their recent study on lamin B1 and chromatin, Shah et al. observed that knocking down lamin B1 expression causes both entry into senescence as well as large scale reorganization of LAD chromatin structure, including regions of H3K4me3 and H3K27me3 marks (Shah et al., 2013).

Age-related changes in nuclear structure have also been described in animal models. In $C$. elegans, nuclear structure undergoes several changes with age, including loss of peripheral heterochromatin and deterioration of nuclear shape and structure. Genetically reducing levels of lamin in the worm also leads to a shortened lifespan (Haithcock et al., 2005). Together these data suggest that breakdown of nuclear architecture is a hallmark of the aging process. However, the details of exactly how spatial organization of chromatin within the nucleus affects regulation of gene expression remain enigmatic. Techniques based on chromosomal conformation capture (3C), including the derivatives $4 \mathrm{C}, 5 \mathrm{C}$, and $\mathrm{Hi}-\mathrm{C}$, have been informative in identifying distal chromatin interactions in a number of organisms. In this family of techniques, chromatin is crosslinked together with formaldehyde, fragmented, and ligated, then examined by a number of methods (most recently high throughput sequencing) for novel ligation products that indicate long range interactions between spatially distinct chromatin domains, representing potentially important regulatory associations (Gibcus and Dekker, 2013). A spatial mapping of the entire human genome using the $\mathrm{Hi}-\mathrm{C}$ technique at $1 \mathrm{mb}$ resolution revealed a segregation of the nucleus into two compartments corresponding to open and closed chromatin (Lieberman-Aiden et al., 2009). The observed chromatin architecture was consistent with a "fractal globule" model where stretches of open or closed chromatin are packed together in dense clusters and organized into larger compartments, with greater interaction within each compartment than across compartments. The S. cerevisiae genome was mapped in a similar manner with $4 \mathrm{C}$, revealing a three dimensional 
structure where the centromeres clustered together at one end of the nucleus, telomeres exhibited both intra- and interchromosomal associations, and rDNA repeats were organized into a distal nucleolus (Duan et al., 2010). Furthermore, interchromosomal interactions were common and include tRNA genes, replication origins, and known sites of chromosomal breakage. Continued use of high resolution techniques such as these will be important for unraveling the complex three dimensional interactions of chromatin, and determining how changes in higher order chromatin organization contribute to aging and age-related phenotypes.

\section{CHROMATIN AND TRANSPOSABLE ELEMENTS}

Heterochromatin establishment and maintenance is important for proper silencing to prevent inappropriate gene expression. One common class of transcripts that could have potentially deleterious effects when expressed is transposable elements (TEs). Most eukaryotic genomes contain a large amount of non-coding DNA that is derived from TEs (reviewed in Slotkin and Martienssen, 2007). For a long time most of these sequences were dismissed as "junk DNA," although in recent years it has become clear that TEs are much more biologically interesting than originally thought, and are in fact widely transcriptionally expressed and can regulate nearby genes (Faulkner et al., 2009). TEs are sequences of DNA that can move within the genome, and are generally divided into two classes: Class I (retrotransposons) and Class II (DNA transposons). The retrotransposon class includes two subgroups: the LTR (long terminal repeat) type, which is flanked by direct repeats, and the non-LTR type, which includes LINEs (long interspersed elements) and SINEs (short interspersed elements). Retrotransposons propagate in a manner similar to retroviruses: they are transcribed by the host cell machinery to an RNA intermediate, encode their own reverse transcriptase which makes a DNA copy of the element, and then are integrated at a new location in the genome. This represents a "copy and paste" mechanism - the old transposon remains in place, and successful retrotransposition results in a new copy and an expanded host genome. DNA transposons, on the other hand, are flanked by inverted repeats, and are excised from the DNA and integrated at a new location by a transposase, which they frequently self encode. DNA transposons thus transpose with a "cut and paste" mechanism that is generally not duplicative, although excision is frequently imperfect and can leave behind partial sequences in the genome. TEs or remnants of TEs make up a large proportion of eukaryotic genomes, generally from 30 to $80 \%$ depending on species (detailed in Kidwell, 2002). The human genome, for example, contains about 50\% TE material, and in some plants the vast majority of the genome consists of TEs (Kidwell and Lisch, 1997; Slotkin and Martienssen, 2007). The relative proportion of different types of TEs also varies appreciably between species. For example, the Drosophila genome has a large number of LTR type retrotransposons, whereas the human genome is characterized by abundant LINEs and SINEs, such as the LINE1 element that makes up 17\% of the genome (Slotkin and Martienssen, 2007).

Most TEs, especially those that have been present in the genome for a very long time, are not active and have accumulated mutations that make them incapable of active transposition
(Faulkner et al., 2009). However, even inactive TEs are still capable of initiating transcription from promoters contained within the element (Conley et al., 2008). TEs are known to be expressed in response to a number of organismal and cellular stresses, including DNA damage, UV, radiation, temperature, wounding, and infection (reviewed in Capy et al., 2000). In addition to DNA damage from radiation, increased concentrations of reactive oxygen species have also been shown to induce expression of TEs (Bradshaw and McEntee, 1989; Ikeda et al., 2001; Stoycheva etal., 2010). A recent study in rats used an acute stress paradigm to examine $\mathrm{H} 3 \mathrm{~K} 9$ methylation and TE expression in the hippocampus (Hunter et al., 2012). In response to stress, H3K9me3 levels increased throughout the hippocampus, but particularly at TE loci, and this was correlated with a repression of expression of certain TEs. TEs are also known to have significant effects on endogenous gene expression, both transcriptionally and post-transcriptionally, through a number of different mechanisms including sense or antisense transcriptional activation, induction of heterochromatic silencing, small RNA targeting, and alternative splicing (reviewed in Feschotte, 2008).

Active, mobile TEs can be highly mutagenic and cause genomic instability, and consequently in order to combat these effects cells have evolved strategies to suppress TE activity. The cellular RNAi machinery prevents TE mobilization by multiple mechanisms (reviewed in Castel and Martienssen, 2013). Details vary by organism and source of RNA, but in general dsRNAs targeted for silencing are processed by Dicer enzymes into short endogenous small interfering RNAs (esiRNAs), and subsequently loaded into an Argonaute (Ago)-containing complex known as RISC (RNA-induced Silencing Complex; reviewed in Pratt and MacRae, 2009). RISC complexes can induce silencing in at least two ways. First, these complexes are known to bind chromatin and repress transcription of their targets by catalyzing formation and spreading of silencing heterochromatin (Verdel et al., 2004; Bühler et al., 2006). This is accomplished by recruiting heterochromatin proteins and histone methyltransferases, resulting in $\mathrm{H} 3 \mathrm{~K} 9$ methylation that is characteristic of transcriptionally silent heterochromatin (Bühler et al., 2006). This mechanism has been well described in yeast, but appears to be widely conserved, including in mammalian cells (reviewed in Sampey et al., 2012). In mammals the siRNA pathway induces transcriptional gene silencing via DNA methylation as well as H3K9 methylation, and this silencing requires both Agol as well as active RNA polymerase II (Morris et al., 2004; Kim et al., 2006; Weinberg et al., 2006). Secondly, in addition to transcriptional gene silencing, RISC complexes perform post-transcriptional silencing of target RNAs by recognizing and hydrolyzing target transcripts, again in a widely conserved mechanism. A catalytically active Ago protein (e.g., Ago2 in humans) binds "guide" siRNAs and "target" RNA transcripts, and subsequently degrades the target with a slicer RNase catalytic activity (Liu et al., 2004; Meister et al., 2004; Gregory et al., 2005; Matranga et al., 2005; Rand et al., 2005). Given the potential mutagenic effects of TE expression and mobilization, these RNAi silencing mechanisms offer an important cellular defense mechanism against genomic damage. 


\section{TRANSPOSABLE ELEMENTS IN AGING}

Because TEs are particularly enriched in heterochromatin, and heterochromatin changes have been linked to the aging process, one attractive hypothesis is that aging may be due in part to increasing inability to control and prevent TE expression and mobilization (Driver and McKechnie, 1992). In fact, because heterochromatin is gene poor, but rich in TEs, TE expression may be the major consequence of the age-related breakdown in heterochromatin structure. A schematic of how this may happen is presented in Figure 1D. In support of this idea, St. Laurent et al. argue in a recent review that transposition of LINE/L1 elements, which are particularly prevalent in mammals and are activated by stress, may play an important role in mammalian aging and evolution ( $\mathrm{St}$ Laurent et al., 2010). In this model, LINE activation leads not only to mutagenic insertions, but also other DNA damage that accumulates with age and can cause genomic instability even in the absence of a successful transposition event. Furthermore, they propose an antagonistic pleiotropy explanation for retrotransposon mobilization, whereby LINE activation provides an evolutionary advantage by allowing for rapid stress-induced genetic variation, but can also lead to reduced individual lifespan as cellular damage accumulates.

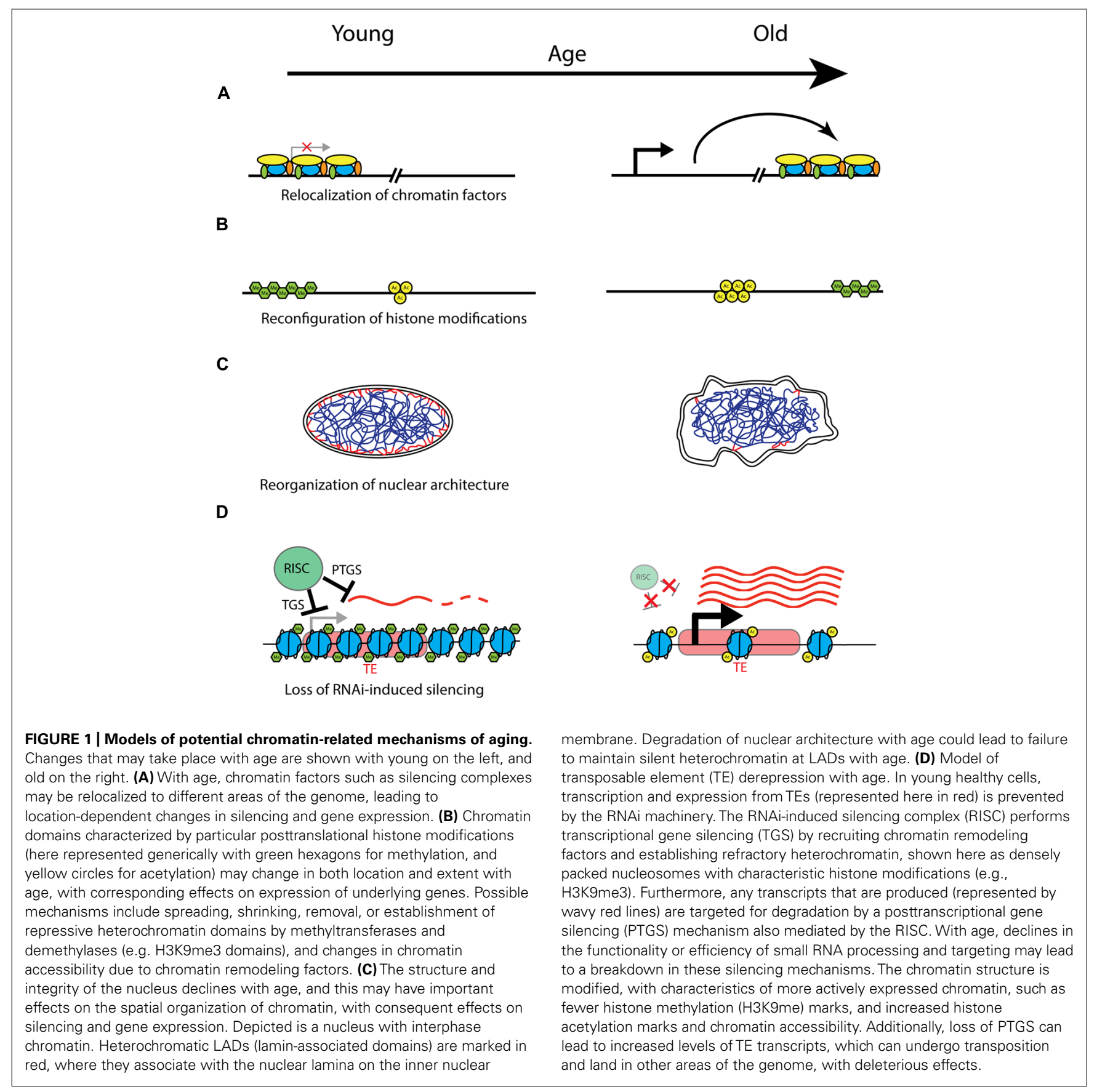


Several recent studies support the involvement of TEs in the aging process. Using a chronological aging model, a study in budding yeast observed that the yeast retrotransposon Tyl showed increased mobility with age, and this was correlated with chromosome rearrangements and other hallmarks of genomic instability (Maxwell et al., 2011). Furthermore, interventions that decreased transposon mobility also exhibited a decrease in age-related deleterious genomic instability. In C. elegans, expression of the endogenous retrotransposon Cer1 (gypsy/Ty3 family) has been shown to increase with age (Dennis et al., 2012). Capsid particles from Cer1 accumulate on microtubules in meiotic germ cells of older animals, and the capsids likely use these microtubules to target host cell nuclei. Retrotransposons are also active in the mammalian nervous system during neural development, and influence neural cell fate by altering expression of neural genes (Muotri et al., 2005). In addition, retrotransposons are also active in several regions of the adult brain, leading to somatic mosaicism (Coufal et al., 2009; Baillie et al., 2011). A recent study examining epigenetic changes in the aging mouse brain observed differences in DNA methylation as well as some histone modifications at repetitive DNA elements with age (Ryu et al., 2011). Specifically, overall histone acetylation of both $\mathrm{H} 3$ and $\mathrm{H} 4$ decreased with age at several TEs as assayed by ChIP-qPCR, and some histone methylation marks, such as $\mathrm{H} 3 \mathrm{~K} 9 \mathrm{me} 3$ and H4K20me3, were also decreased in some TEs.

Transposable elements have also been implicated in cellular senescence. As human adult stem cells undergo senescence, DNA damage becomes concentrated in hot spots within TEs, and there is a noted increase in transcription of Alu elements (Wang et al., 2011; De Cecco et al., 2013). When Alu transcripts were targeted for degradation in these cells by shRNA, the cells were able to reverse senescent phenotypes and begin proliferating, demonstrating that TE expression can cause cellular toxicity (Wang et al., 2011). De Cecco et al. (2013) observed a number of TEs that were enriched in late stage senescent cells when examined with FAIRE-seq (which measures chromatin accessibility), including not only Alu elements but also L1, SVA, and satellite sequences. Interestingly, the evolutionarily more recent L1 elements, which are more likely to be capable of retrotransposition, showed the highest enrichment in late senescent cells. In accordance with the observed increase in FAIRE-seq signal of L1 elements, both RNA levels as well as genomic copy number increased in senescent cells, suggesting mobilization and transposition of these elements during senescence. Additionally, a pair of recent studies examined the role of Alu elements and Dicer1 function in age-related macular degeneration (Kaneko et al., 2011; Tarallo et al., 2012). Knocking down Dicer1, which processes Alu transcripts for degradation, resulted in higher than normal Alu expression in retinal pigmented epithelium (RPE) cells, and this Alu RNA was cytotoxic, leading to RPE degeneration and disease progression (Kaneko et al., 2011). Furthermore, inhibition of the Alu RNAs by antisense oligos reversed this cytotoxic phenotype.

The adult Drosophila brain also shows retrotransposon activity in certain mushroom body neurons, and this is correlated with a relative lack of the piRNA proteins Aubergine and Argonaute 3, which normally function to suppress TE activity post-transcriptionally in the germline (Perrat et al., 2013). This study provided evidence that the piRNA pathway may also be active in somatic tissue, and its disruption was associated with increased somatic TE expression. TE activation has also been linked to aging in Drosophila. Li et al. (2013) showed that expression of several TEs increases with age in the fly brain, and using a GFP engineered gypsy element (the gypsy-TRAP reporter) were able to detect somatic transposition that increased with age in the brain. Finally, disrupting the RNAi protein Ago2 caused both an increase in transposition as well as a shortening of lifespan and impairment of memory with age (Li et al., 2013). It is important to note that because of the central role of Argonaute proteins in a host of small RNA pathways, Ago depletion also leads to many effects not directly related to TE silencing, including defects in microRNA processing, development, and differentiation (O'Carroll et al., 2007; Su et al., 2009; Shekar et al., 2011). These studies examining TE regulation with age, again in multiple model systems, suggest that changes in TE expression and transposition may play an important role in the cellular dysfunction observed during organismal aging.

\section{CONCLUSION}

A number of different lines of evidence now point to chromatin structure, and heterochromatin in particular, as being an important modulator of aging (summarized in Table 1). A common thread throughout many theories of aging holds that the functional decline associated with aging results from an inability to properly maintain cellular structure and function with time. The chromatin context, including nucleosome structure, histone modifications, and nuclear spatial organization, has a large influence over gene expression, and evidence is accumulating that failure to maintain proper chromatin structure may be a driver of the increased damage and disruption of homeostasis associated with aging. The chromatin changes observed with age are multifaceted, and not always consistent across species and model systems, or even cell types. For instance, as mentioned above, although numerous animal model systems have shown a decrease in both overall histone as well as heterochromatin abundance with age, one of the most prominent chromatin phenotypes of the replicative senescence model is the accumulation of heterochromatin foci with age. This suggests that the role of chromatin structure in aging is likely more complex than the earlier heterochromatin loss model of aging would suggest. There are a number of potentially relevant chromatin changes that may underlie the aging process (presented in Figure 1). In addition to the age-related histone loss observed in yeast and mammalian replicative senescence (Feser et al., 2010; O'Sullivan et al., 2010), components of chromatin may redistribute to other locations in the genome with age, causing changes in gene expression and/or genomic stability (Figure 1A). An example of this mechanism is the age-related loss of heterochromatic silencing in yeast, with concomitant relocalization of the SIR silencing complex away from telomeres and mating type loci and toward rDNA (Kennedy et al., 1997). A similar mechanism operates in mammalian cells, where SIRT1 redistributes within the genome in response to DNA damage, with consequent changes in gene expression that parallel those seen in aging (Oberdoerffer et al., 2008). Chromatin domains containing characteristic histone acetylation or methylation marks may also spread, diminish, 
Table 1 | Summary of major findings of recent literature linking aging and chromatin.

\begin{tabular}{|c|c|c|}
\hline Organism & Summary of observations & Reference \\
\hline S. cerevisiae & Increased H4K16ac, decreased Sir2, loss of histones with age & Dang et al. (2009) \\
\hline S. cerevisiae & Loss of histones with age. Overexpression of histones extends lifespan & Feser etal. (2010) \\
\hline Human cells & $\begin{array}{l}\text { Formation during senescence of senescence-associated heterochromatin foci, with high } \\
\text { levels of H3K9me3, H3K27me3, and HP1 }\end{array}$ & $\begin{array}{l}\text { Narita et al. (2003), Zhang et al. (2005), } \\
\text { Chandra et al. (2012) }\end{array}$ \\
\hline Human cells & $\begin{array}{l}\text { Enrichment in } \mathrm{H} 3 \mathrm{~K} 4 \mathrm{me} 3 \text { and } \mathrm{H} 3 \mathrm{~K} 27 \mathrm{me} 3 \text { marks in LADs with age. Loss of H3K4me3 at } \\
\text { downregulated genes, loss of } \mathrm{H} 3 \mathrm{~K} 27 \mathrm{me} 3 \text { at upregulated genes }\end{array}$ & Shah et al. (2013) \\
\hline Human cells & $\begin{array}{l}\text { Smoothing of chromatin-open and closed regions more similar to each other during } \\
\text { senescence. Increased expression of numerous TEs }\end{array}$ & De Cecco et al. (2013) \\
\hline C. elegans & $\begin{array}{l}\text { H3K4 methyltransferase knockdown increases lifespan. H3K4 demethylase knockdown } \\
\text { shortens lifespan }\end{array}$ & Greer et al. (2010, 2011) \\
\hline C. elegans & LSD1 knockdown (H3K4 and H3K9 demethylase) increases lifespan & McColl et al. (2008) \\
\hline C. elegans & $\begin{array}{l}\text { UTX-1 knockdown (H3K27 demethylase) increases lifespan. Decline in H3K27 methylation } \\
\text { with age }\end{array}$ & Jin etal. (2011), Maures et al. (2011) \\
\hline D. melanogaster & H3K4me3, H3K36me3 decline with age. H3K9me3, HP1 increase and relocalize with age & Wood et al., 2010 \\
\hline D. melanogaster & Decreased heterochromatin with age, HP1 overexpression extends lifespan & Larson et al. (2012) \\
\hline D. melanogaster & H3K27 methyltransferase knockdown increases lifespan & Siebold et al. (2010) \\
\hline Mouse & Numerous tissue-specific changes in DNA methylation patterns with age & Maegawa et al. (2010) \\
\hline Mouse & Decreased histone acetylation, increased histone methylation with age in cochlea & Watanabe and Bloch (2013) \\
\hline Mouse & $\begin{array}{l}\text { Histone methylation and acetylation changes correlate with increased memory formation } \\
\text { in neurodegeneration model }\end{array}$ & Fischer et al. (2007) \\
\hline Mouse & H4K12 hypoacetylation with age in hippocampus. HDAC inhibitor improves phenotypes & Peleg et al. (2010) \\
\hline Rat & $\begin{array}{l}\text { Reduced H3K9 and H4K12 acetylation with age correlates with LTP defect. HDAC inhibitor } \\
\text { improves phenotypes }\end{array}$ & Zeng et al. (2011) \\
\hline Mouse & $\begin{array}{l}\text { Decreased H4 acetylation in Alzheimer's model with LTP defects. HDAC inhibitor improves } \\
\text { phenotypes }\end{array}$ & Francis etal. (2009), Kilgore etal. (2010) \\
\hline Mouse & $\begin{array}{l}\text { H4K16 hypoacetylation in progeria model, HDAC inhibition improves phenotypes and } \\
\text { extends lifespan }\end{array}$ & Krishnan etal. (2011) \\
\hline Mouse cells & Gene expression correlates with nuclear lamina colocalization & Peric-Hupkes et al. (2010) \\
\hline Human cells & Nuclear lamin B1 decreases during senescence & Shimi etal. (2011), Freund etal. (2012) \\
\hline Human cells & Defects in lamin A processing and nuclear structure with age & $\begin{array}{l}\text { Scaffidi and Misteli (2006), } \\
\text { McClintock et al. (2007) }\end{array}$ \\
\hline C. elegans & $\begin{array}{l}\text { Loss of peripheral heterochromatin and deterioration of nuclear structure with age. Lamin } \\
\text { knockdown shortens Ifespan }\end{array}$ & Haithcock et al. (2005) \\
\hline Rat & Acute stress induces $\mathrm{H} 3 \mathrm{~K} 9 \mathrm{me} 3$ and TE silencing in hippocampus & Hunter etal. (2012) \\
\hline S. cerevisiae & Ty1 mobility and genomic instability increase with age & Maxwell et al. (2011) \\
\hline C. elegans & Cer1 retrotransposon expression increased with age & Dennis etal. (2012) \\
\hline Mouse & DNA methylation and histone modifications (Ac/Me) change at TE loci with age & Ryu et al. (2011) \\
\hline Human cells & $\begin{array}{l}\text { Alu transcripts increase during senescence, targeting by shRNA reverses senescent } \\
\text { phenotype }\end{array}$ & Wang etal. (2011) \\
\hline Human, mouse & $\begin{array}{l}\text { Dicer1 loss in age-related macular degeneration leads to increased cytotoxic Alu } \\
\text { transcripts, antisense targeting of Alu reverses phenotypes }\end{array}$ & Kaneko etal. (2011) \\
\hline D. melanogaster & Retrotransposons are active in fly neurons & Perrat et al. (2013) \\
\hline D. melanogaster & $\begin{array}{l}\text { TE expression and somatic transposition increases with age in fly brain. Disrupting RNAi } \\
\text { pathway causes increase in transposition and memory defects }\end{array}$ & Li et al. (2013) \\
\hline
\end{tabular}


redistribute or rearrange with age, with the local chromatin and genomic context determining the phenotypic outcome of such changes (Figure 1B). Examples of this mechanism are prevalent at a genomic scale in worm, fly, and mammalian studies as referenced above, although the downstream effects of such rearrangements remain largely undescribed. Changes in spatial organization of various chromatin domains within the nucleus, association, or dissociation of heterochromatin with the nuclear lamina, and breakdowns in nuclear architecture and structure are also likely to be important hallmarks of aging (Figure 1C). The prevalence and localization of histone variants may also change with age, and this altered nucleosome composition can influence silencing and gene expression. Given the increasingly recognized role of the RNAi machinery in establishment and maintenance of heterochromatin and silencing, this pathway represents yet another potential mechanism of age-related dysfunction (Figure 1D). Declines in the efficiency of small RNA processing, proper genomic targeting of RNAi, and establishment and maintenance of RNAi-based transcriptional and posttranscriptional silencing all represent potential mechanisms for aging phenotypes. Finally, of particular interest is a potential role for mobilization of retrotransposons and other TEs in age-related phenotypes. TEs are widely spread throughout most eukaryotic genomes, and are known to be regulated by cellular heterochromatinization and the RNAi pathway, making this an attractive explanation for how age-related breakdown in homeostasis in these regulatory pathways may lead to deleterious mutations and genomic instability. It is likely that the phenotypes of aging are influenced by more than one of these pathways, and different mechanisms may be acting on different locations in the genome or in different cell and tissue types. Unraveling the complexity of these mechanisms and the importance of the local chromatin and genomic context will be key to understanding the downstream effects of chromatin reorganization on the aging process.

In future experiments it will be increasingly important to move on from exploring associations between chromatin structure and age and toward a causal mechanism of specifically how observed chromatin changes lead to a decline in cellular homeostasis. It is likely that increased use of genetic tools, including heterochromatin and TE-based reporters, in model systems will yield mechanistic insights into the relationship between chromatin structure and aging. In addition, the decreasing cost and increasing availability of next generation sequencing-based techniques provides the opportunity to query the localization of histone modifications, longer range chromatin interactions, and the transcription of non-canonical RNAs at the whole genome level to unravel the influence of these epigenetic effects on the aging process.

\section{ACKNOWLEDGMENTS}

The authors wish to thank Suzanne Hosier and Brian Jones for providing critical readings and content suggestions. This work was supported by Ellison Foundation/AFAR Awards to Jason G. Wood and NIA grants AG16667, AG24353 and AG25277 to Stephen L. Helfand. Stephen L. Helfand is an Ellison Medical Research Foundation Senior Investigator and recipient of a Glenn Award for Research in Biological Mechanisms of Aging.

\section{REFERENCES}

Andrulis, E. D., Neiman, A. M., Zappulla, D. C., and Sternglanz, R. (1998). Perinuclear localization of chromatin facilitates transcriptional silencing. Nature 394, 592-595. doi: 10.1038/29100

Baillie, J. K., Barnett, M. W., Upton, K. R., Gerhardt, D. J., Richmond, T. A., De Sapio, F., et al. (2011). Somatic retrotransposition alters the genetic landscape of the human brain. Nature 479, 534-537. doi: 10.1038/nature10531

Bickmore, W. A., and van Steensel, B. (2013). Genome architecture: domain organization of interphase chromosomes. Cell 152, 1270-1284. doi: 10.1016/j.cell.2013.02.001

Bradshaw, V. A., and McEntee, K. (1989). DNA damage activates transcription and transposition of yeast Ty retrotransposons. Mol. Gen. Genet. 218, 465-474. doi: 10.1007/BF00332411

Bühler, M., Verdel, A., and Moazed, D. (2006). Tethering RITS to a nascent transcript initiates RNAi- and heterochromatin-dependent gene silencing. Cell 125, 873886. doi: 10.1016/j.cell.2006.04.025

Capy, P., Gasperi, G., Biemont, C., and Bazin, C. (2000). Stress and transposable elements: co-evolution or useful parasites? Heredity (Edinb.) 85, 101-106. doi: 10.1046/j.1365-2540.2000.00751.x

Castel, S. E., and Martienssen, R. A. (2013). RNA interference in the nucleus: roles for small RNAs in transcription, epigenetics and beyond. Nat. Rev. Genet. 14, 100-112. doi: 10.1038/nrg3355

Chandra, T., Kirschner, K., Thuret, J.-Y., Pope, B. D., Ryba, T., Newman, S., et al. (2012). Independence of repressive histone marks and chromatin compaction during senescent heterochromatic layer formation. Mol. Cell 47, 203-214. doi: 10.1016/j.molcel.2012.06.010

Conley, A., Miller, W., and Jordan, I. (2008). Human cis natural antisense transcripts initiated by transposable elements. Trends Genet. 24, 53-56. doi: 10.1016/j.tig.2007.11.008

Coufal, N. G., Garcia-Perez, J. L., Peng, G. E., Yeo, G. W., Mu, Y., Lovci, M. T., et al. (2009). L1 retrotransposition in human neural progenitor cells. Nature 460, 1127-1131. doi: 10.1038/nature08248

Dang, W., Steffen, K. K., Perry, R., Dorsey, J. A., Johnson, F. B., Shilatifard, A., et al. (2009). Histone H4 lysine 16 acetylation regulates cellular lifespan. Nature 459, 802-807. doi: 10.1038/nature08085

De Cecco, M., Criscione, S. W., Peckham, E. J., Hillenmeyer, S., Hamm, E. A., Manivannan, J., etal. (2013). Genomes of replicatively senescent cells undergo global epigenetic changes leading to gene silencing and activation of transposable elements. Aging Cell 12, 247-256. doi: 10.1111/acel. 12047

Dennis, S., Sheth, U., Feldman, J. L., English, K. A., and Priess, J. R. (2012). C. elegans germ cells show temperature and age-dependent expression of Cer1, a Gypsy/Ty3-related retrotransposon. PLoS Pathog. 8:e1002591. doi: 10.1371/journal.ppat.1002591

Driver, C. J., and McKechnie, S. W. (1992). Transposable elements as a factor in the aging of Drosophila melanogaster. Ann. N. Y. Acad. Sci. 673, 83-91. doi: 10.1111/j.1749-6632.1992.tb27439.x

Duan, Z., Andronescu, M., Schutz, K., McIlwain, S., Kim, Y. J., Lee, C., et al. (2010). A three-dimensional model of the yeast genome. Nature 465, 363-367. doi: 10.1038/nature08973

Eriksson, M., Brown, W. T., Gordon, L. B., Glynn, M. W., Singer, J., Scott, L., et al. (2003). Recurrent de novo point mutations in lamin A cause HutchinsonGilford progeria syndrome. Nature 423, 293-298. doi: 10.1038/nature 01629

Faulkner, G. J., Kimura, Y., Daub, C. O., Wani, S., Plessy, C., Irvine, K. M., et al. (2009). The regulated retrotransposon transcriptome of mammalian cells. Nat. Genet. 41, 563-571. doi: 10.1038/ng.368

Feschotte, C. (2008). Transposable elements and the evolution of regulatory networks. Nat. Rev. Genet. 9, 397-405. doi: 10.1038/nrg2337

Feser, J., Truong, D., Das, C., Carson, J. J., Kieft, J., Harkness, T., et al. (2010). Elevated histone expression promotes life span extension. Mol. Cell 39, 724-735. doi: 10.1016/j.molcel.2010.08.015

Feser, J., and Tyler, J. (2011). Chromatin structure as a mediator of aging. FEBS Lett. 585, 2041-2048. doi: 10.1016/j.febslet.2010.11.016

Feuerbach, F., Galy, V., Trelles-Sticken, E., Fromont-Racine, M., Jacquier, A., Gilson, E., et al. (2002). Nuclear architecture and spatial positioning help establish transcriptional states of telomeres in yeast. Nat. Cell Biol. 4, 214-221. doi: $10.1038 /$ ncb756 
Fischer, A., Sananbenesi, F., Wang, X., Dobbin, M., and Tsai, L.-H. (2007). Recovery of learning and memory is associated with chromatin remodelling. Nature 447, 178-182. doi: 10.1038/nature05772

Francis, Y. I., Fà, M., Ashraf, H., Zhang, H., Staniszewski, A., Latchman, D. S., et al. (2009). Dysregulation of histone acetylation in the APP/PS1 mouse model of Alzheimer's disease. J. Alzheimers Dis. 18, 131-139.

Frankel, S., and Rogina, B. (2005). Drosophila longevity is not affected by heterochromatin mediated gene silencing. Aging Cell 4, 53-56. doi: 10.1111/j.14749726.2005.00143.x

Fraser, P., and Bickmore, W. (2007). Nuclear organization of the genome and the potential for gene regulation. Nature 447, 413-417. doi: 10.1038/nature05916

Freund, A., Laberge, R.-M., Demaria, M., and Campisi, J. (2012). Lamin B1 loss is a senescence-associated biomarker. Mol. Biol. Cell 23, 2066-2075. doi: 10.1091/mbc.E11-10-0884

Frye, R. A. (2000). Phylogenetic classification of prokaryotic and eukaryotic Sir2-like proteins. Biochem. Biophys. Res. Commun. 273, 793-798. doi: $10.1006 /$ bbrc. 2000.3000

Gibcus, J. H., and Dekker, J. (2013). The hierarchy of the 3D genome. Mol. Cell 49, 773-782. doi: 10.1016/j.molcel.2013.02.011

Giresi, P. G., and Lieb, J. D. (2009). Isolation of active regulatory elements from eukaryotic chromatin using FAIRE (formaldehyde assisted isolation of regulatory elements). Methods 48, 233-239. doi: 10.1016/j.ymeth.2009.03.003

Goldman, R. D., Shumaker, D. K., Erdos, M. R., Eriksson, M., Goldman, A. E., Gordon, L. B., et al. (2004). Accumulation of mutant lamin A causes progressive changes in nuclear architecture in Hutchinson-Gilford progeria syndrome. Proc. Natl. Acad. Sci. U.S.A. 101, 8963-8968. doi: 10.1073/pnas.0402943101

Gotta, M., Laroche, T., Formenton, A., Maillet, L., Scherthan, H., and Gasser, S. M. (1996). The clustering of telomeres and colocalization with Rap1, Sir3, and Sir4 proteins in wild-type Saccharomyces cerevisiae. J. Cell Biol. 134.

Greer, E. L., Maures, T. J., Hauswirth, A. G., Green, E. M., Leeman, D. S., Maro, G. S., et al. (2010). Members of the H3K4 trimethylation complex regulate lifespan in a germline-dependent manner in C. elegans. Nature 466, 383-387. doi: 10.1038/nature09195

Greer, E. L., Maures, T. J., Ucar, D., Hauswirth, A. G., Mancini, E., Lim, J. P., et al. (2011). Transgenerational epigenetic inheritance of longevity in Caenorhabditis elegans. Nature 479, 365-371. doi: 10.1038/nature10572

Gregory, R. I., Chendrimada, T. P., Cooch, N., and Shiekhattar, R. (2005). Human RISC couples microRNA biogenesis and posttranscriptional gene silencing. Cell 123, 631-640. doi: 10.1016/j.cell.2005.10.022

Guelen, L., Pagie, L., Brasset, E., Meuleman, W., Faza, M. B., Talhout, W., et al. (2008). Domain organization of human chromosomes revealed by mapping of nuclear lamina interactions. Nature 453, 948-951. doi: 10.1038/nature06947

Haigis, M. C., and Sinclair, D. A. (2010). Mammalian sirtuins: biological insights and disease relevance. Annu. Rev. Pathol. 5, 253-295. doi: 10.1146/annurev.pathol.4.110807.092250

Haithcock, E., Dayani, Y., Neufeld, E., Zahand, A. J., Feinstein, N., Mattout, A., et al. (2005). Age-related changes of nuclear architecture in Caenorhabditis elegans. Proc. Natl. Acad. Sci. U.S.A. 102, 16690-16695. doi: 10.1073/pnas.0506955102

Hunter, R. G., Murakami, G., Dewell, S., Seligsohn, M., Baker, M. E. R., Datson, N. A., et al. (2012). Acute stress and hippocampal histone $\mathrm{H} 3$ lysine 9 trimethylation, a retrotransposon silencing response. Proc. Natl. Acad. Sci. U.S.A. 109, 1765717662. doi: 10.1073/pnas.1215810109

Ikeda, K., Nakayashiki, H., Takagi, M., Tosa, Y., and Mayama, S. (2001). Heat shock, copper sulfate and oxidative stress activate the retrotransposon MAGGY resident in the plant pathogenic fungus Magnaporthe grisea. Mol. Genet. Genomics 266, 318-325. doi: 10.1007/s004380100560

Imai, S., Armstrong, C. M., Kaeberlein, M., and Guarente, L. (2000). Transcriptional silencing and longevity protein Sir2 is an NAD-dependent histone deacetylase. Nature 403, 795-800. doi: 10.1038/35001622

Ivanov, A., Pawlikowski, J., Manoharan, I., van Tuyn, J., Nelson, D. M., Rai, T. S., et al. (2013). Lysosome-mediated processing of chromatin in senescence. J. Cell Biol. 202, 129-143. doi: 10.1083/jcb.201212110

Jin, C., Li, J., Green, C. D., Yu, X., Tang, X., Han, D., et al. (2011). Histone demethylase UTX-1 regulates $C$. elegans life span by targeting the insulin/IGF-1 signaling pathway. Cell Metab. 14, 161-172. doi: 10.1016/j.cmet.2011.07.001

Kaeberlein, M., McVey, M., and Guarente, L. (1999). The SIR2/3/4 complex and SIR2 alone promote longevity in Saccharomyces cerevisiae by two different mechanisms. Genes Dev. 13, 2570-2580. doi: 10.1101/gad.13.19.2570
Kaneko, H., Dridi, S., Tarallo, V., Gelfand, B. D., Fowler, B. J., Cho, W. G., et al. (2011). DICER1 deficit induces Alu RNA toxicity in age-related macular degeneration. Nature 471, 325-330. doi: 10.1038/nature09830

Kennedy, B. K., Austriaco, N. R., Zhang, J., and Guarente, L. (1995). Mutation in the silencing gene SIR4 can delay aging in S. cerevisiae. Cell 80, 485-496. doi: 10.1016/0092-8674(95)90499-9

Kennedy, B. K., Gotta, M., Sinclair, D. A., Mills, K., McNabb, D. S., Murthy, M., et al. (1997). Redistribution of silencing proteins from telomeres to the nucleolus is associated with extension of life span in S. cerevisiae. Cell 89, 381-391. doi: 10.1016/S0092-8674(00)80219-6

Kidwell, M. G. (2002). Transposable elements and the evolution of genome size in eukaryotes. Genetica 115, 49-63. doi: 10.1023/A:1016072014259

Kidwell, M. G., and Lisch, D. (1997). Transposable elements as sources of variation in animals and plants. Proc. Natl. Acad. Sci. U.S.A. 94, 7704-7711. doi: 10.1073/pnas.94.15.7704

Kilgore, M., Miller, C. A., Fass, D. M., Hennig, K. M., Haggarty, S. J., Sweatt, J. D., et al. (2010). Inhibitors of class 1 histone deacetylases reverse contextual memory deficits in a mouse model of Alzheimer's disease. Neuropsychopharmacology 35, 870-880. doi: 10.1038/npp.2009.197

Kim, D. H., Villeneuve, L. M., Morris, K. V., and Rossi, J. J. (2006). Argonaute-1 directs siRNA-mediated transcriptional gene silencing in human cells. Nat. Struct. Mol. Biol. 13, 793-797. doi: 10.1038/nsmb1142

Kim, S., Villeponteau, B., and Jazwinski, S. M. (1996). Effect of replicative age on transcriptional silencing near telomeres in Saccharomyces cerevisiae. Biochem. Biophys. Res. Commun. 219, 370-376. doi: 10.1006/bbrc.1996.0240

Kosar, M., Bartkova, J., Hubackova, S., Hodny, Z., Lukas, J., and Bartek, J. (2011). Senescence-associated heterochromatin foci are dispensable for cellular senescence, occur in a cell type- and insult-dependent manner and follow expression of p16 ink4a. Cell Cycle 10, 457-468. doi: 10.4161/cc.10.3.14707

Krishnan, V., Chow, M. Z. Y., Wang, Z., Zhang, L., Liu, B., Liu, X., et al. (2011). Histone $\mathrm{H} 4$ lysine 16 hypoacetylation is associated with defective DNA repair and premature senescence in Zmpste24-deficient mice. Proc. Natl. Acad. Sci. U.S.A. 108, 12325-12330. doi: 10.1073/pnas.1102789108

Landry, J., Sutton, A., Tafrov, S. T., Heller, R. C., Stebbins, J., Pillus, L., et al. (2000). The silencing protein SIR2 and its homologs are NAD-dependent protein deacetylases. Proc. Natl. Acad. Sci. U.S.A. 97, 5807-5811. doi: 10.1073/pnas.1101 48297

Larson, K., Yan, S.-J., Tsurumi, A., Liu, J., Zhou, J., Gaur, K., et al. (2012). Heterochromatin formation promotes longevity and represses ribosomal RNA synthesis. PLoS Genet. 8:e1002473. doi: 10.1371/journal.pgen. 1002473

Li, W., Prazak, L., Chatterjee, N., Grüninger, S., Krug, L., Theodorou, D., et al. (2013). Activation of transposable elements during aging and neuronal decline in Drosophila. Nat. Neurosci. 16, 529-531. doi: 10.1038/nn.3368

Lieberman-Aiden, E., van Berkum, N. L., Williams, L., Imakaev, M., Ragoczy, T., Telling, A., et al. (2009). Comprehensive mapping of long-range interactions reveals folding principles of the human genome. Science 326, 289-293. doi: 10.1126/science.1181369

Liu, J., Carmell, M. A., Rivas, F. V., Marsden, C. G., Thomson, J. M., Song, J.-J., et al. (2004). Argonaute2 is the catalytic engine of mammalian RNAi. Science 305 , 1437-1441. doi: 10.1126/science.1102513

Macieira-Coelho, A. (1991). Chromatin reorganization during senescence of proliferating cells. Mutat. Res. 256, 81-104. doi: 10.1016/0921-8734(91)90003-T

Maegawa, S., Hinkal, G., Kim, H. S., Shen, L., Zhang, L., Zhang, J., et al. (2010). Widespread and tissue specific age-related DNA methylation changes in mice. Genome Res. 20, 332-340. doi: 10.1101/gr.096826.109

Matranga, C., Tomari, Y., Shin, C., Bartel, D. P., and Zamore, P. D. (2005). Passengerstrand cleavage facilitates assembly of siRNA into Ago2-containing RNAi enzyme complexes. Cell 123, 607-620. doi: 10.1016/j.cell.2005.08.044

Maures, T. J., Greer, E. L., Hauswirth, A. G., and Brunet, A. (2011). The H3K27 demethylase UTX-1 regulates C. elegans lifespan in a germline-independent, insulin-dependent manner. Aging Cell 10, 980-990. doi: 10.1111/j.14749726.2011.00738.x

Maxwell, P. H., Burhans, W. C., and Curcio, M. J. (2011). Retrotransposition is associated with genome instability during chronological aging. Proc. Natl. Acad. Sci. U.S.A. 108, 20376-20381. doi: 10.1073/pnas.1100271108

McClintock, D., Ratner, D., Lokuge, M., Owens, D. M., Gordon, L. B., Collins, F. S., et al. (2007). The mutant form of lamin A that causes Hutchinson-Gilford 
progeria is a biomarker of cellular aging in human skin. PLoS ONE 2:e1269. doi: 10.1371/journal.pone.0001269

McColl, G., Killilea, D. W., Hubbard, A. E., Vantipalli, M. C., Melov, S., and Lithgow, G. J. (2008). Pharmacogenetic analysis of lithium-induced delayed aging in Caenorhabditis elegans. J. Biol. Chem. 283, 350-357. doi: 10.1074/jbc.M705028200

Meister, G., Landthaler, M., Patkaniowska, A., Dorsett, Y., Teng, G., and Tuschl, T. (2004). Human argonaute 2 mediates RNA cleavage targeted by miRNAs and siRNAs. Mol. Cell 15, 185-197. doi: 10.1016/j.molcel.2004. 07.007

Millar, C. B., and Grunstein, M. (2006). Genome-wide patterns of histone modifications in yeast. Nat. Rev. Mol. Cell Biol. 7, 657-666. doi: 10.1038/ nrm1986

Morris, K. V., Chan, S. W.-L., Jacobsen, S. E., and Looney, D. J. (2004). Small interfering RNA-induced transcriptional gene silencing in human cells. Science 305, 1289-1292. doi: 10.1126/science.1101372

Muotri, A. R., Chu, V. T., Marchetto, M. C. N., Deng, W., Moran, J. V., and Gage, F. H. (2005). Somatic mosaicism in neuronal precursor cells mediated by L1 retrotransposition. Nature 435, 903-910. doi: 10.1038/nature 03663

Narita, M., Nun, S., Heard, E., Narita, M., Lin, A. W., Hearn, S. A., et al. (2003). Rb-mediated heterochromatin formation and silencing of E2F target genes during cellular senescence. Cell 113, 703-716. doi: 10.1016/S0092-8674(03) 00401-X

O'Carroll, D., Mecklenbrauker, I., Das, P. P., Santana, A., Koenig, U., Enright, A. J., et al. (2007). A Slicer-independent role for argonaute 2 in hematopoiesis and the microRNA pathway. Genes Dev. 21, 1999-2004. doi: 10.1101/gad. 1565607

O'Sullivan, R. J., and Karlseder, J. (2012). The great unravelling: chromatin as a modulator of the aging process. Trends Biochem. Sci. 37, 466-476. doi: 10.1016/j.tibs.2012.08.001

O'Sullivan, R. J., Kubicek, S., Schreiber, S. L., and Karlseder, J. (2010). Reduced histone biosynthesis and chromatin changes arising from a damage signal at telomeres. Nat. Struct. Mol. Biol. 17, 1218-1225. doi: 10.1038/nsmb.1897

Oberdoerffer, P., Michan, S., McVay, M., Mostoslavsky, R., Vann, J., Park, S. K., et al. (2008). SIRT1 redistribution on chromatin promotes genomic stability but alters gene expression during aging. Cell 135, 907-918. doi: 10.1016/j.cell.2008.10.025

Oberdoerffer, P., and Sinclair, D. A. (2007). The role of nuclear architecture in genomic instability and ageing. Nat. Rev. Mol. Cell Biol. 8, 692-702. doi: $10.1038 / \mathrm{nrm} 2238$

Peleg, S., Sananbenesi, F., Zovoilis, A., Burkhardt, S., Bahari-Javan, S., Agis-Balboa, R. C., et al. (2010). Altered histone acetylation is associated with age-dependent memory impairment in mice. Science 328, 753-757. doi: 10.1126/science. 1186088

Peric-Hupkes, D., Meuleman, W., Pagie, L., Bruggeman, S. W. M., Solovei, I., Brugman, W., et al. (2010). Molecular maps of the reorganization of genomenuclear lamina interactions during differentiation. Mol. Cell 38, 603-613. doi: 10.1016/j.molcel.2010.03.016

Perrat, P. N., DasGupta, S., Wang, J., Theurkauf, W., Weng, Z., Rosbash, M., et al. (2013). Transposition-driven genomic heterogeneity in the Drosophila brain. Science 340, 91-95. doi: 10.1126/science.1231965

Pickersgill, H., Kalverda, B., de Wit, E., Talhout, W., Fornerod, M., and van Steensel, B. (2006). Characterization of the Drosophila melanogaster genome at the nuclear lamina. Nat. Genet. 38, 1005-1014. doi: 10.1038/ng1852

Pratt, A. J., and MacRae, I. J. (2009). The RNA-induced silencing complex: a versatile gene-silencing machine. J. Biol. Chem. 284, 17897-17901. doi: 10.1074/jbc.R900012200

Rand, T. A., Petersen, S., Du, F., and Wang, X. (2005). Argonaute2 cleaves the anti-guide strand of siRNA during RISC activation. Cell 123, 621-629. doi: 10.1016/j.cell.2005.10.020

Rieder, D., Trajanoski, Z., and McNally, J. G. (2012). Transcription factories. Front. Genet. 3:221. doi: 10.3389/fgene.2012.00221

Ryu, S. H., Kang, K., Yoo, T., Joe, C. O., and Chung, J. H. (2011). Transcriptional repression of repeat-derived transcripts correlates with histone hypoacetylation at repetitive DNA elements in aged mice brain. Exp. Gerontol. 46, 811-818. doi: 10.1016/j.exger.2011.07.001

Sampey, G. C., Guendel, I., Das, R., Jaworski, E., Klase, Z., Narayanan, A., et al. (2012). Transcriptional gene silencing (TGS) via the RNAi machinery in HIV-1 infections. Biology 1, 339-369. doi: 10.3390/biology1020339
Scaffidi, P., and Misteli, T. (2006). Lamin A-dependent nuclear defects in human aging. Science 312, 1059-1063. doi: 10.1126/science.1127168

Shah, P. P., Donahue, G., Otte, G. L., Capell, B. C., Nelson, D. M., Cao, K., et al. (2013). Lamin B1 depletion in senescent cells triggers large-scale changes in gene expression and the chromatin landscape. Genes Dev. 27, 1787-1799. doi: $10.1101 /$ gad.223834.113

Shekar, P. C., Naim, A., Sarathi, D. P., and Kumar, S. (2011). Argonaute-2-null embryonic stem cells are retarded in self-renewal and differentiation. J. Biosci. 36, 649-657. doi: 10.1007/s12038-011-9094-1

Shimi, T., Butin-Israeli, V., Adam, S. A., Hamanaka, R. B., Goldman, A. E., Lucas, C. A., et al. (2011). The role of nuclear lamin B1 in cell proliferation and senescence. Genes Dev. 25, 2579-2593. doi: 10.1101/gad.179515.111

Siebold, A. P., Banerjee, R., Tie, F., Kiss, D. L., Moskowitz, J., and Harte, P. J. (2010). Polycomb repressive complex 2 and trithorax modulate Drosophila longevity and stress resistance. Proc. Natl. Acad. Sci. U.S.A. 107, 169-174. doi: 10.1073/pnas.0907739107

Slotkin, R. K., and Martienssen, R. (2007). Transposable elements and the epigenetic regulation of the genome. Nat. Rev. Genet. 8, 272-285. doi: 10.1038/nrg2072

Smeal, T., Claus, J., Kennedy, B., Cole, F., and Guarente, L. (1996). Loss of transcriptional silencing causes sterility in old mother cells of S. cerevisiae. Cell 84, 633-642. doi: 10.1016/S0092-8674(00)81038-7

St Laurent, G., Hammell, N., and McCaffrey, T. A. (2010). A LINE-1 component to human aging: do LINE elements exact a longevity cost for evolutionary advantage? Mech. Ageing Dev. 131, 299-305. doi: 10.1016/j.mad.2010. 03.008

Stoycheva, T., Pesheva, M., and Venkov, P. (2010). The role of reactive oxygen species in the induction of Ty1 retrotransposition in Saccharomyces cerevisiae. Yeast 27, 259-267. doi: 10.1002/yea.1749

Su, H., Trombly, M. I., Chen, J., and Wang, X. (2009). Essential and overlapping functions for mammalian argonautes in microRNA silencing. Genes Dev. 23, 304-317. doi: 10.1101/gad.1749809

Tarallo, V., Hirano, Y., Gelfand, B. D., Dridi, S., Kerur, N., Kim, Y., et al. (2012). DICER1 loss and Alu RNA induce age-related macular degeneration via the NLRP3 inflammasome and MyD88. Cell 149, 847-859. doi: 10.1016/j.cell.2012.03.036

Tsurumi, A., Li, W. X., and Changes, E. (2012). Global heterochromatin loss: a unifying theory of aging? Epigenetics 7, 680-688. doi: 10.4161/epi.20540

Verdel, A., Jia, S., Gerber, S., Sugiyama, T., Gygi, S., Grewal, S. I. S., et al. (2004). RNAi-mediated targeting of heterochromatin by the RITS complex. Science 303, 672-676. doi: 10.1126/science. 1093686

Villeponteau, B. (1997). The heterochromatin loss model of aging. Exp. Gerontol. 32, 383-394. doi: 10.1016/S0531-5565(96)00155-6

Wang, J., Geesman, G. J., Hostikka, S. L., Atallah, M., Blackwell, B., Lee, E., et al. (2011). Inhibition of activated pericentromeric SINE/Alu repeat transcription in senescent human adult stem cells reinstates self-renewal. Cell Cycle 10, 3016-3030. doi: $10.4161 /$ cc. 10.17 .17543

Watanabe, K.-I., and Bloch, W. (2013). Histone methylation and acetylation indicates epigenetic change in the aged cochlea of mice. Eur. Arch. Otorhinolaryngol. 270, 1823-1830. doi: 10.1007/s00405-012-2222-1

Weinberg, M. S., Villeneuve, L. M., Ehsani, A. L. I., Amarzguioui, M., Aagaard, L., Chen, Z., et al. (2006). The antisense strand of small interfering RNAs directs histone methylation and transcriptional gene silencing in human cells. RNA 12, 256-262. doi: 10.1261/rna.2235106

Wood, J. G., Hillenmeyer, S., Lawrence, C., Chang, C., Hosier, S., Lightfoot, W., et al. (2010). Chromatin remodeling in the aging genome of Drosophila. Aging Cell 9, 971-978. doi: 10.1111/j.1474-9726.2010.00624.x

Zeng, Y., Tan, M., Kohyama, J., Sneddon, M., Watson, J. B., Sun, Y. E., et al. (2011). Epigenetic enhancement of BDNF signaling rescues synaptic plasticity in aging. J. Neurosci. 31, 17800-17810. doi: 10.1523/JNEUROSCI.3878-11. 2011

Zhang, R., Poustovoitov, M. V., Ye, X., Santos, H. A., Chen, W., Daganzo, S. M., et al. (2005). Formation of MacroH2A-containing senescence-associated heterochromatin foci and senescence driven by ASFla and HIRA. Dev. Cell 8, 19-30. doi: 10.1016/j.devcel.2004.10.019

Conflict of Interest Statement: The authors declare that the research was conducted in the absence of any commercial or financial relationships that could be construed as a potential conflict of interest. 
Received: 09 October 2013; accepted: 18 November 2013; published online: 04 December 2013.

Citation: Wood JG and Helfand SL (2013) Chromatin structure and transposable elements in organismal aging. Front. Genet. 4:274. doi: 10.3389/fgene.2013.00274

This article was submitted to Genetics of Aging, a section of the journal Frontiers in Genetics.
Copyright (C) 2013 Wood and Helfand. This is an open-access article distributed under the terms of the Creative Commons Attribution License (CC BY). The use, distribution or reproduction in other forums is permitted, provided the original author (s) or licensor are credited and that the original publication in this journal is cited, in accordance with accepted academic practice. No use, distribution or reproduction is permitted which does not comply with these terms. 